\title{
Single photon emission computed tomography-computed tomography visualization of sentinel lymph nodes for lymph flow guided nodal irradiation in oral tongue cancer
}

\author{
Sergey Nikolaevich Novikov', Pavel Ivanovich Krzhivitskii' ${ }^{1}$ Zamira Achmedovna Radgabova ${ }^{2}$, \\ Maxim Andreevitch Kotov', Mikhail Markovich Girshovich', Anna Sergeevna Artemyeva ${ }^{3}$, \\ Yulia Sergeevna Melnik', Sergey Vasilevich Kanaev ${ }^{1}$ \\ ${ }^{1}$ Department of Radiation Oncology \& Nuclear Medicine, N.N. Petrov National Medical Research Center of Oncology, St. Petersburg, Russia \\ ${ }^{2}$ Department of Head and Neck Surgery, N.N. Petrov National Medical Research Center of Oncology, St Petersburg, Russia \\ ${ }^{3}$ Department of Pathology, N.N. Petrov National Medical Research Center of Oncology, St Petersburg, Russia
}

Received: March 25, 2021

Revised: May 25, 2021

Accepted: June 9, 2021

\section{Correspondence:}

Sergey Nikolaevich Novikov Department of Radiation Oncology \& Nuclear Medicine, N.N. Petrov National Medical Research Center of Oncology, Leningradskaya, 68, St. Petersburg 197758, Russia

Tel: +79500437996

E-mail:krokon@mail.ru

ORCID:

https://orcid.org/0000-0002-7185-1967
Purpose: To evaluate correlation of single photon emission computed tomography-computed tomography (SPECT-CT) data on lymph flow (LF) from oral tongue cancer (OC) and the topography of lymph nodes (LN) metastases; to determine the clinical value of lymph flow guided radiotherapy (LFGRT). Materials and Methods: SPECT-CT visualization of LF from the OC lesions was performed after peritumoral injection of ${ }^{99 \mathrm{~m}} \mathrm{Tc}$-phytate in 26 primary patients with clinical stage cT1-2NOMO disease. We determined the individual drainage (unilateral/bilateral) from the tumor, and localization of sentinel LNs according to the neck levels. Metastases in LNs were verified with histology and a 2-year follow-up.

Results: SPECT-CT detected bilateral LF in 10 (38.5\%) of 26 patients; in 16 (61.5\%) cases the drainage was unilateral. Histology revealed LNs metastases in three cases; regional recurrences were diagnosed in other four patients. In all seven observations metastases were located at the same site and level as the sentinel LNs. In eight (30.8\%) of 26 patients sentinel LNs were visualized unilaterally at levels Ib-Ila; in five cases, unilaterally at levels I-Ila-III. In these patients, LFGRT demonstrated 59\%$70 \%$ reduction of irradiated volume, and $26 \%-42 \%$ and $51 \%-70 \%$ decrease of the mean dose to the spinal cord and the contralateral parotid gland. In patients with a bilateral drainage the reduction of doses absorbed by the spinal cord and contralateral parotid gland was 19\% and 6\%, respectively. Conclusion: Localization of sentinel LNs determined by SPECT-CT corresponds to the localization of metastatic LNs in terms of side and levels.

Keywords: Oral tongue cancer, Sentinel lymph nodes, Lymph flow, Radiotherapy

\section{Introduction}

The results of prospective randomized studies in patients with oral cancer demonstrated that a standard bilateral irradiation of the neck provided a significant increase of overall and relapse-free survival $[1,2]$. The opinion on the required and sufficient volumes to be irradiated in different clinical situations is less definitive. The cur- rent guidelines recommend a bilateral neck irradiation with exceptions in special cases only, as in case of unilateral tonsil involvement when regional radiotherapy can be performed only on the lymph nodes (LNs) ipsilateral to the primary lesion. Unfortunately, the available imaging modalities are not enough accurate to identify patients with a low risk of LNs involvement: sensitivity of ultrasound, magnetic resonance imaging (MRI), positron emission to- 
mography-computed tomography (PET-CT) in diagnosing subclinical metastatic lesions does not exceed 46\%-80\% [3-6]. Meanwhile, the reduction of neck LN irradiation volumes including unilateral radiotherapy in patients with oropharyngeal malignancies is of utmost importance as the minimization of the irradiation volume is associated with sparing normal tissues and a significant reduction in frequency and grade of early and late toxicity of the treatment $[7,8]$.

The concept of sentinel lymph node (SLN) appeared in the last quarter of the 20th century, and was successfully implemented in the routine treatment of patients with breast cancer and melanoma. The main idea of this concept is based on the assumption of the sequential and predictable manner of lymph flow from the tumor to the regional LNs, and hence on predictable order of LNs metastatic involvement [9]. The logical evolution of these ideas led to the concept of lymph flow guided radiotherapy already discussed in patients with breast, prostate, head and neck, and cervical cancer [10-13]. The main purpose of the present study was to evaluate the correlation of single photon emission computed tomography-computed tomography (SPECT-CT) data on lymph flow from oral tongue cancer and the topography of LNs metastases revealed with histological examinations and during the follow-up. We analyze the value of this information for lymph flow guided radiotherapy in patients with oral tongue cancer.

\section{Materials and Methods}

This retrospective study approved by the Institutional Ethic Committee of the N.N. Petrov National Medical Research Cancer Center (No. 20). Finally, 26 primary patients with stage I-II (cT1$2 \mathrm{NOMO}$ ) carcinoma of oral tongue were included in the analysis. In all cases the primary staging, treatment and follow-up were performed at the Institute from January 2015 to November 2019. The baseline characteristics of the study group are presented in Table 1.

All patients had clinically non-involved LNs confirmed by clinical examination, ultrasound of the neck, contrast-enhanced CT and MRI. The main signs of LN metastases were: a short axis diameter of more than $8 \mathrm{~mm}$, a round shape, presence of necrosis, irregularity of the borders/margins, and change of cortex thickness and/or loss of fatty hilus.

For the SLN mapping we used ${ }^{99 \mathrm{~m}} \mathrm{Tc}$-radiocolloids ( ${ }^{99 \mathrm{~m}} \mathrm{Tc}$-phytate) with the diameter ranging from $80-100 \mathrm{~nm}$ to $1,000 \mathrm{~nm}$. The hybrid SPECT-CT visualization of sentinel and second echelon LNs with tracer uptake was performed 120-240 minutes after the peritumoral injection of ${ }^{99 \mathrm{~m}} \mathrm{Tc}$-phytate $(0.2-0.4 \mathrm{~mL})$ in four points around the primary lesion (3,6, 9, and 12 o'clock positions). The
Table 1. Patient demographics and tumor characteristics $(n=26)$

\begin{tabular}{lc}
\hline Characteristic & Value \\
\hline Sex & $14(53.8)$ \\
Male & $12(42.2)$ \\
Female & $55.6(33-84)$ \\
Age (yr) & \\
Lateralized primary tumor & $5(19.2)$ \\
No & $21(80.8)$ \\
Yes & \\
T stage & $14(53.8)$ \\
T1 & $12(42.6)$ \\
T2 & \\
Grade & $9(34.6)$ \\
G1 & $15(57.7)$ \\
G2 & $2(7.7)$ \\
G3 & \\
Lymph flow (SPECT-CT) & $16(61.5)$ \\
Unilateral & $10(38.5)$ \\
Bilateral & \\
Neck dissection & $2(7.7)$ \\
Radical (I-V levels) & $24(92.3)$ \\
Elective (I-III levels) & \\
\hline &
\end{tabular}

Values are presented as number (\%) or median (range). SPECT-CT, single photon emission computed tomography-computed tomography.

total injected activity ranged from $100 \mathrm{MBq}$ to $150 \mathrm{MBq}$. The patients were positioned supine on the table in the treatment position. The acquisition started with the following parameters: $128 \times$ 128 matrix; scan step, $3^{\circ}$; shot exposure time, 16 seconds; rotation angle for each detector, $180^{\circ}$. Upon the completion of the SPECT, a spiral CT was performed without changing the patient's position on the table. All LNs with uptake of ${ }^{99 \mathrm{~m}} \mathrm{Tc}$-phytate were categorized as sentinel LNs according to the recent approach in patients with head and neck cancer [14].

The SPECT-CT data were analyzed by an experienced ( 10 years of practice) radiologist and a radiation oncologist. They assessed the lymph flow pattern (unilateral or bilateral), number and topography of SLNs. The visualized SLNs were anatomically categorized according to the levels of the neck as proposed by the consensus guidelines for the delineation of the neck node levels [15]. The next day after the SLN mapping all patients underwent surgery with SLN biopsy followed by a unilateral LNs dissection on the site of the tumor. This strategy was approved by the Institute Tumor Board and considered as a routine treatment approach. The extent of LNs dissection was not directed by the topography of visualized SLNs: in two cases we performed "radical unilateral neck LNs dissection" (dissections of LNs localized at the I-V levels); in the remaining 24 cases, "elective" LNs dissection (dissection of LNs from levels I-III). 
In all cases sentinel LNs identified with the gamma-probe and non-sentinel (without radioactivity) LNs from all anatomic levels were separately sent for a pathological examination.

The pathological examination of SLNs was performed according to the protocol recommended by the SENT study [16]. Nodes of less than $2 \mathrm{~mm}$ were examined as a whole; nodes of 2-5 $\mathrm{mm}$ were cut into 2 halves along the greatest distance from the LN poles. LNs of more than $5 \mathrm{~mm}$ were sliced into $2 \mathrm{~mm}$ sections. Cross-sections were stained with hematoxylin and eosin (H\&E) and examined with a light microscopy. In case of non-metastatic SLNs additional staining with pancytocreatin AE1/AE3 antibodies was performed. Non-sentinel LNs were examined according to the standard protocol: single representative section of each half of the node was stained by H\&E for a final light microscopy. Based on histopathology results, metastases were differentiated into isolated tumor cells $(<0.2 \mathrm{~mm})$, micrometastases $(0.2-2 \mathrm{~mm}$ ) or macrometastases ( $>2 \mathrm{~mm}$ ).

At least a 2-year follow-up upon the completion of the treatment was reached for all patients. The schedule of the visits was as follows: every 3 months during the first year and every 6 months during the subsequent period. For the first 2 years, the follow-up procedures included physical examinations, MRI and ultrasound scanning.

Finally, with the Eclipse 3D treatment planning system (Varian, Santa Clara, CA, USA), we compared virtual radiotherapy plans prepared for a standard radiotherapy of neck LNs and primary lesion according to existing guidelines and experimental plans for lymph flow guided radiotherapy when irradiation volumes were restricted by the primary lesion and neck levels with SLNs. To compare the treatment plans we used the following parameters: clinical treatment volume (in $\mathrm{cm}^{3}$ ), the mean whole body dose, the mean dose absorbed by the irradiated spine and the mean dose to the ipsilateral and contralateral parotid gland.

\section{Reference test and statistical analysis}

The purpose of the study was to evaluate the correlation of SPECTCT data on lymph flow from oral tongue cancer and topography of regional LNs metastases revealed with histopathology and/or during follow-up. All patients underwent the unilateral neck dissection on the site of primary lesion even in cases when primary tumor was close to the median sulcus. Histopathologic examination of excised LNs and the follow-up data were both used as a reference. Patients with positive SLNs and/or non-SLNs localized at the same levels as SNLs were considered true positive. Also, all regional recurrences corresponding to the levels containing SLNs at the primary SPECT-CT were considered as true positive. All cases with lymph node metastases and/or regional recurrences detected distally from the levels containing SLNs were considered as false negative. These data were used to calculate the negative predictive value and sensitivity of SPECT-CT data on lymph flow in predicting topography of neck metastases.

\section{Results}

SLNs were successfully visualized in all 26 primary patients included in the study. All patients had SLNs identified during the surgery. We found a close correlation between the localization of the primary lesion and the pattern of lymph flow from the tumor: unilateral or bilateral (Fig. 1). SPECT-CT demonstrated bilateral localization of LNs with uptake of ${ }^{99 \mathrm{~m}} \mathrm{Tc}$-phytate in 10 (38.5\%) cases. This pattern was detected in four of five patients with a medial localization of the primary lesion. At the same time, six of 21 (28.6\%) patients with lateralized lesions had SLNs visualized on both sides of the neck.

Other 16 (61.5\%) patients had a unilateral drainage from the primary lesion that manifested in the unilateral localization of radioactive LNs on the side of the tumor. We did not detect any case of the unilateral lymph flow to the contralateral side of the neck. Interestingly, in 15 of 16 patients with the unilateral lymph flow pattern primary lesions had a lateral localization.

The pathological examination of the removed neck LNs revealed metastases in three of 26 patients included into the study. All three cases were classified as micrometastases. In two patients metastases were localized in SLNs without tumor involvement of nonSLNs, and in the last case metastases were diagnosed in non-SLNs localized in the neck level containing SLNs.

It is important to note that pathological examinations of the removed LNs and a median 29 months follow-up confirmed that none of 16 patients with the unilateral lymph flow had a contralateral metastatic LN involvement and/or contralateral recurrences. On the contrary, one of 10 patients with the bilateral localization of SLNs determined with the SPECT-CT examination had a progression in contralateral SLNs. During the initial treatment, the patient with a primary lateralized lesion had a unilateral LNs dissection only on the side of primary tumor. During the pathological examination no metastases were identified in sentinel and non-SLNs, and it was decided to refrain from the contralateral neck dissection.

The median follow-up for 26 evaluated patients was 29 months (range, 24 to 56 months). During this follow-up period, we diagnosed four regional recurrences: in one patient with the bilateral lymph flow who had a unilateral LNs dissection, tumor progression was detected in a SLN localized on the contralateral side of the neck. This SLN was successfully visualized with SPECT-CT performed before the primary surgery. Despite the findings of SPECT$\mathrm{CT}$ the unilateral neck/cervical LNs dissection was performed due 

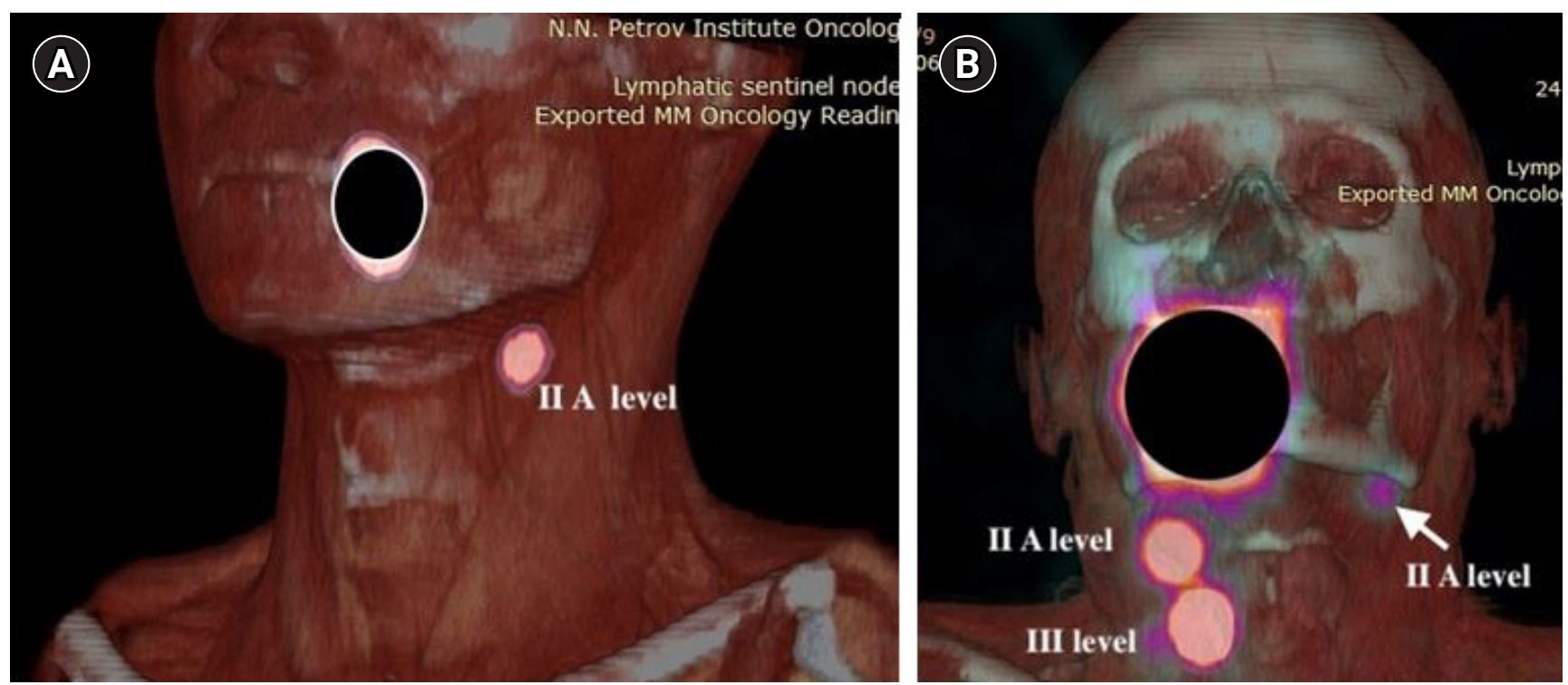

Fig. 1. SPECT-CT visualization of lymph flow from oral tongue cancer. (A) The identification of unilateral lymphatic drainage of the tongue cancer (injection site is masked). Three-dimensional volume rendered SPECT-CT image revealing that cervical sentinel lymph node is located in the Ila level. (B) The identification of bilateral lymphatic drainage of the tongue cancer (injection site is masked). Three-dimensional volume rendered SPECT-CT image revealing that cervical sentinel lymph nodes are located in the lla level on the left side (arrow) and Ila-III levels on the right side. SPECT-CT, single photon emission computed tomography-computed tomography.

to the presence of a lateralized primary tumor. In other three non-irradiated patients the neck recurrences were revealed at the level with SLNs present: level la in one, level III in other two observations. Distant lung metastases were detected in 2 (7.7\%) cases. To the date of the last follow-up, one patient with lung metastases died from the oral tongue generalization 14 months after the treatment started.

Thus, the survival rate was $96.2 \%$, the disease-free survival rate $76.9 \%$, and locoregional recurrence rate $15.4 \%$.

Finally, we found that in six of seven cases neck metastases diagnosed with the pathomorphological examination or revealed as recurrences during the follow-up were localized in SLNs or in nonSLNs on the levels bearing "hot" LNs. In one case, metastatic LN was revealed on the level proximal to the level bearing radioactive LNs. The histopathologic findings and the follow-up data indicate high sensitivity (100\%) and negative predictive value (100\%) of SPECT-CT Iymph flow visualization when it is used to predict localization of oral tongue metastases according to the neck site and levels. Tables 2 and 3 show the rate and distribution of SLNs detected with SPECT-CT. In nine (34.6\%) of 26 patients SLNs were visualized only at levels I-II (Tables 2, 3); whereby in eight of nine mentioned cases the lymph flow was unilateral.

Since the study group was mostly represented by patients with an early oral tongue cancer, only three men received adjuvant radiotherapy: chemoradiotherapy in patient with T2 oral tongue cancer and a positive margin; radiotherapy-in patient with lympho-
Table 2. Localization of SLNs and LN metastases in patients with unilateral lymph flow from primary tumor

\begin{tabular}{|c|c|c|c|c|}
\hline No. & $\begin{array}{l}\text { Distance from } \\
\text { the tumor edge } \\
\text { to median } \\
\text { sulcus }(\mathrm{mm})\end{array}$ & $\begin{array}{l}\text { Ipsilateral levels } \\
\text { with SLNs }\end{array}$ & $\begin{array}{l}\text { Ipsilateral } \\
\text { levels with } \\
\text { metastatic } \\
\text { LNs }\end{array}$ & $\begin{array}{l}\text { Ipsilateral } \\
\text { levels with } \\
\text { recurrent LNs }\end{array}$ \\
\hline 1 & 12 & Ila-III & - & - \\
\hline 2 & 4 & Ila & - & - \\
\hline 3 & - & Ila-Ilb & - & - \\
\hline 4 & 5 & Ila & Ila non-SLN & - \\
\hline 5 & 1 & Ila-IIb-III-IV & III SLN & - \\
\hline 6 & 5 & Ib-Ila-I|b-III-IV & - & - \\
\hline 7 & 5 & Ila-Ilb-III-IV & - & - \\
\hline 8 & 5 & la & - & - \\
\hline 9 & 9 & |b-||a-||b-III & - & III \\
\hline 10 & 1 & Ilb & - & - \\
\hline 11 & 1 & \|la-Ilb-III & Ilb SLN & - \\
\hline 12 & 13 & IIa-IIb-III & - & III \\
\hline 13 & 16 & Ila & - & - \\
\hline 14 & 18 & Ila & - & - \\
\hline 15 & 7 & Ila & - & - \\
\hline 16 & 5 & Ilb-III & - & - \\
\hline
\end{tabular}

SLN, sentinel lymph node; LN, lymph node.

vascular invasion and another one-with close margin. Therefore, we evaluated the potential of lymph flow guided strategy of definitive or adjuvant radiotherapy/chemoradiotherapy in reduction of normal tissue irradiation according to three clinical models. The 
Table 3. Localization of SLNs and LN metastases in patients with bilateral lymph flow from primary tumor

\begin{tabular}{|c|c|c|c|c|c|}
\hline No. & $\begin{array}{l}\text { Distance from the tumor } \\
\text { edge to median sulcus }(\mathrm{mm})\end{array}$ & $\begin{array}{l}\text { Ipsilateral levels with } \\
\text { SLNs }\end{array}$ & $\begin{array}{l}\text { Contralateral levels } \\
\text { with SLNs }\end{array}$ & $\begin{array}{l}\text { Ipsilateral levels with } \\
\text { metastatic LNs }\end{array}$ & $\begin{array}{l}\text { Levels with recurrent } \\
\text { LNs }\end{array}$ \\
\hline 1 & 8 & Ila-Ilb-III & IIa-III & No & No \\
\hline 2 & 6 & Ilb & Ilb & No & la non-SLN ipsilatera \\
\hline 3 & 6 & Ila-III & Ila-III & No & Ila SLN contralateral \\
\hline 4 & 9 & |a-IIa-III & Ila-III & No & No \\
\hline 5 & 6 & Ila & III & No & No \\
\hline 6 & 0 & Ila-III & Ila-III & No & No \\
\hline 7 & 0 & $\|b-\| I \mid$ & Ilb & No & No \\
\hline 8 & 0 & III & III & No & No \\
\hline 9 & 6 & Ila-III & III & No & No \\
\hline 10 & 0 & IIa-III & Ila & No & No \\
\hline
\end{tabular}

SLN, sentinel lymph node; LN, lymph node.
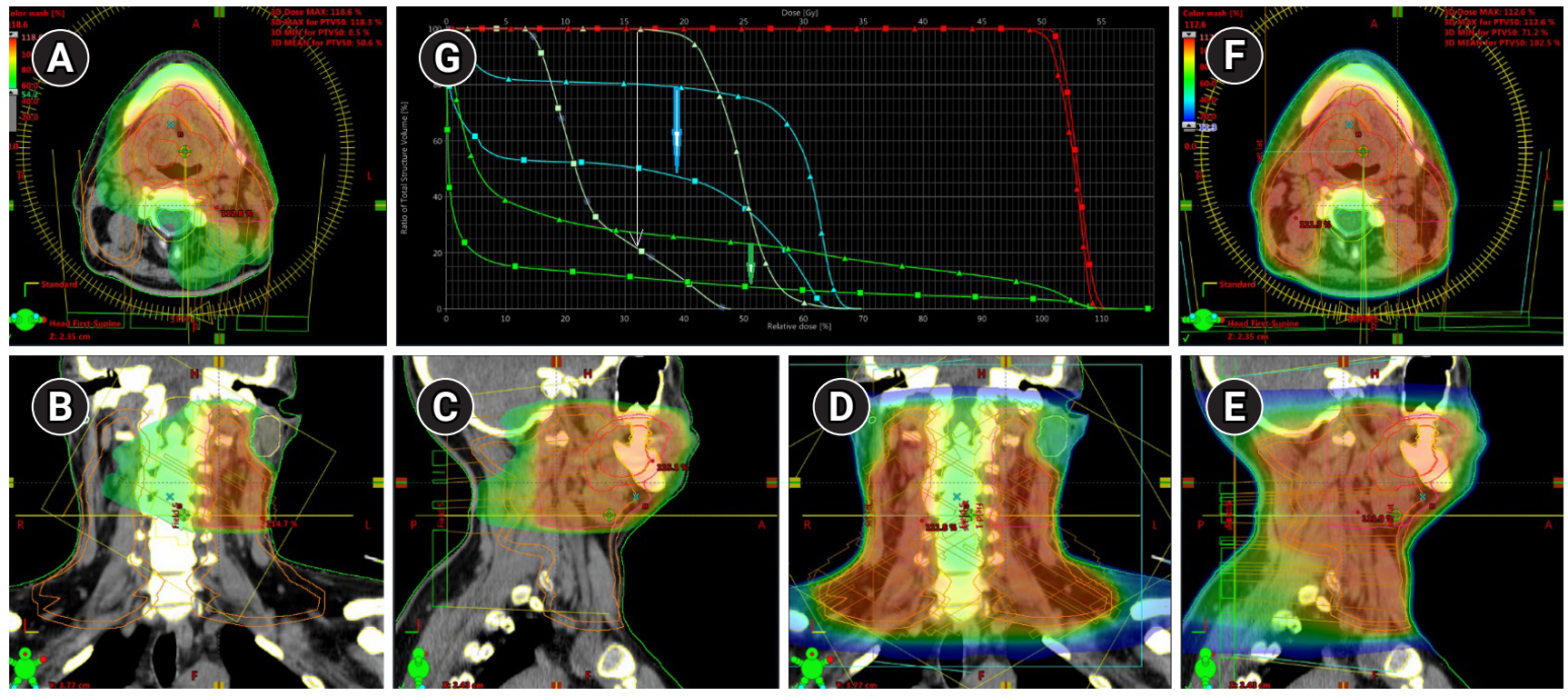

Fig. 2. Treatment plan (dose distribution) for "lymph flow guided" radiotherapy of patient $\mathrm{G}$ with a unilateral localization of sentinel lymph nodes on the levels I-Ila in (A) axial, (B) coronal, and (C) sagittal projections. Dose distribution for standard bilateral irradiation of neck lymph nodes presented in (D) axial, (E) coronal, and (F) sagittal projections. (G) Analysis of dose volume histograms demonstrated a significant advantage of lymph flow directed irradiation confirmed with a dramatic reduction of the doses absorbed by cervical spinal cord (blue arrow), contralateral parotid gland (white arrow) with simultaneous reduction of the total amount of irradiated tissues (green lines).

first was represented by the patient with the unilateral lymph flow to the I-Ila neck levels, the second by the unilateral lymph flow to levels I-III, and the third by the bilateral lymph flow. For each model we created plans for a standard bilateral elective LN irradiation, and a plan for lymph flow guided radiotherapy.

The first model was represented by patient $K$. who had the unilateral lymph flow to levels I-Ila. In this case, the clinical treatment volume could be decreased by 70\%: from 1,224 $\mathrm{cm}^{3}$ (for a standard bilateral elective LNs irradiation) to $367 \mathrm{~cm}^{3}$ (for a lymph flow guided strategy); the mean dose absorbed by the cervical spinal cord could be reduced from 24.7 to $14.3 \mathrm{~Gy}(42 \%)$; the mean dose to the contralateral parotid gland from 24.6 to 12.4 Gy (51\%) (Fig. 2).

In 14 (53.8\%) of 26 cases, the SPECT-CT data demonstrated that SLNs were localized at level III and proximally; whereby in five of those 14 cases the lymph flow pattern was unilateral. In addition, a very similar pattern of unilateral lymph flow to the LNs localized on levels I-IV was detected in other three patients. As an example, the comparative analysis of the standard and "lymph flow guided" radiotherapy plans of patient $P$. showed that with the unilateral irradiation of LNs located at the levels I-III (model II) the clinical treatment volume decreased by 59\%: from 1,209 to $485 \mathrm{~cm}^{3}$. This was associated with $26 \%$ and $70 \%$ reduction of mean doses ab- 
sorbed by the cervical spinal cord and contralateral parotid gland from 23.5 to 17.4 Gy and from 23.2 to 6.9 Gy, respectively (Fig. 3).

In cases when SLNs were visualized at levels I-III on the involvement side and at the II level of the contralateral side of the neck (model III), the comparative analysis of the standard and "lymph flow guided" radiotherapy plans shows that the clinical treatment volume could be decreased by $37 \%$ from 629 to $397 \mathrm{~cm}^{3}$. It was associated with 19\% and 6\% reduction of mean doses absorbed by the cervical spinal cord and contralateral parotid gland from 26.9 to $21.3 \mathrm{~Gy}$ and from 27.7 to $26.1 \mathrm{~Gy}$, respectively (Fig. 4).

\section{Discussion and Conclusion}

In the present study we found out that the topography of SLNs could effectively predict localizations of metastatic LNs and hence be used for lymph flow guided radiotherapy. In particular, our results demonstrate that $61.5 \%$ of patients with oral tongue cancer had a unilateral lymph flow to cervical LNs. Moreover, in patients with lateralized primary lesions the unilateral SPECT-CT Iocalization of "hot" LNs was mentioned in $73.2 \%$ cases. In the SUSPECT study [8], the authors also revealed the prevalence (in 80\% cases) of the unilateral lymph flow in patients with lesions not crossed by the median sulcus of the tongue.

According to data obtained by Farmer et al. [17], a unilateral lymph flow from the oral carcinoma was detected in 107 of 140 patients (76\% cases). It may be assumed that patients with a unilater- al lymph flow pattern are subjects to a unilateral LNs dissection and/or cervical LNs irradiation only on the side of the visualized LNs. The approach mentioned above is supported by the data obtained by the EORTC study [18]. Literature analysis performed by AlMamgani et al. [7] reports that after a unilateral LNs dissection in 1,116 patients with head and neck cancer, contralateral LNs recurrences were found only in 2.4\% of cases. However, this number increased to $12.1 \%$ in case of a medial lesion. According to Singh et al. [19], the frequency of contralateral metastases in patients with tumor crossing midline can reach $29 \%$. This can be explained by a high frequency of a bilateral lymph flow in this category of patients. Our results indicate that $38.5 \%$ of the evaluated patients with oral tongue cancer had SPECT-CT signs of a bilateral lymphatic drainage into the cervical LNs, whereas in $40 \%$ of those cases the primary tumor was localized medially, crossing the median sulcus.

Thus, our results and the literature data indicate that the bilateral LNs dissection and/or irradiation must be considered in patients with a bilateral lymph flow pattern. On the contrary, the presence of a unilateral lymphatic drainage suggests the opportunity for unilateral treatment interventions. In addition, we revealed that in all cases LN metastases detected pathologically or revealed during the follow-up were localized proximally or at the same levels as "hot" LNs detected by SPECT-CT examination (as an additional argument in favor of a lymph flow guided treatment strategy).

The results of $3 \mathrm{D}$ modeling demonstrate that according to the concept of lymph flow guided radiotherapy in case of a unilateral
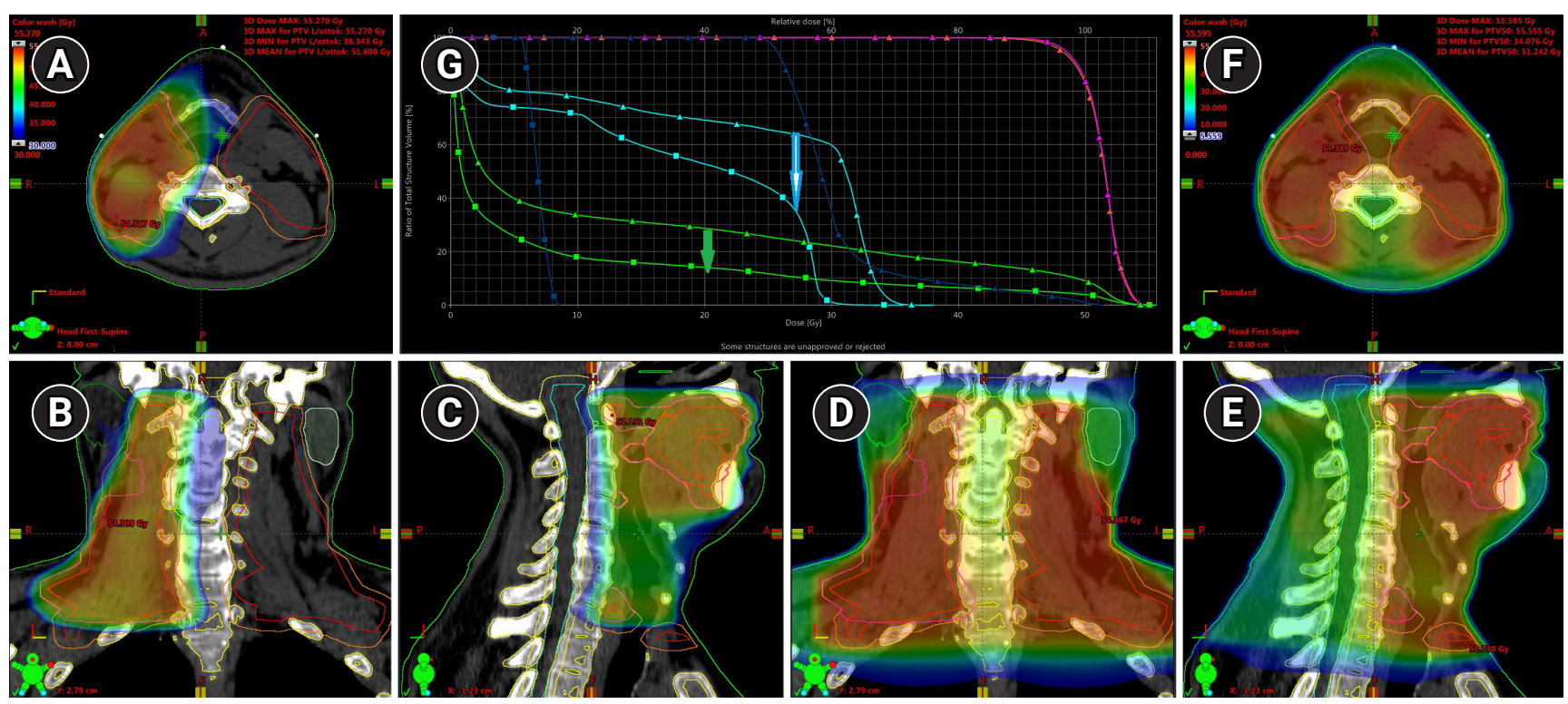

Fig. 3. Treatment plan for "lymph flow guided" radiotherapy of patient $P$ with a unilateral localization of "hot" lymph nodes (with uptake of $99 m$ Tc-phytate) on levels I-IV dose distribution in (A) axial, (B) coronal, and (C) sagittal projections. Dose distribution for standard bilateral irradiation of neck lymph nodes presented in (D) axial, (E) coronal, and (F) sagittal projections. (G) Analysis of dose volume histograms demonstrated a significant reduction of the doses absorbed by contralateral parotid gland (white arrow, blue lines), cervical spinal cord (blue arrow) with a simultaneous reduction of the total amount of irradiated tissues (green lines). 

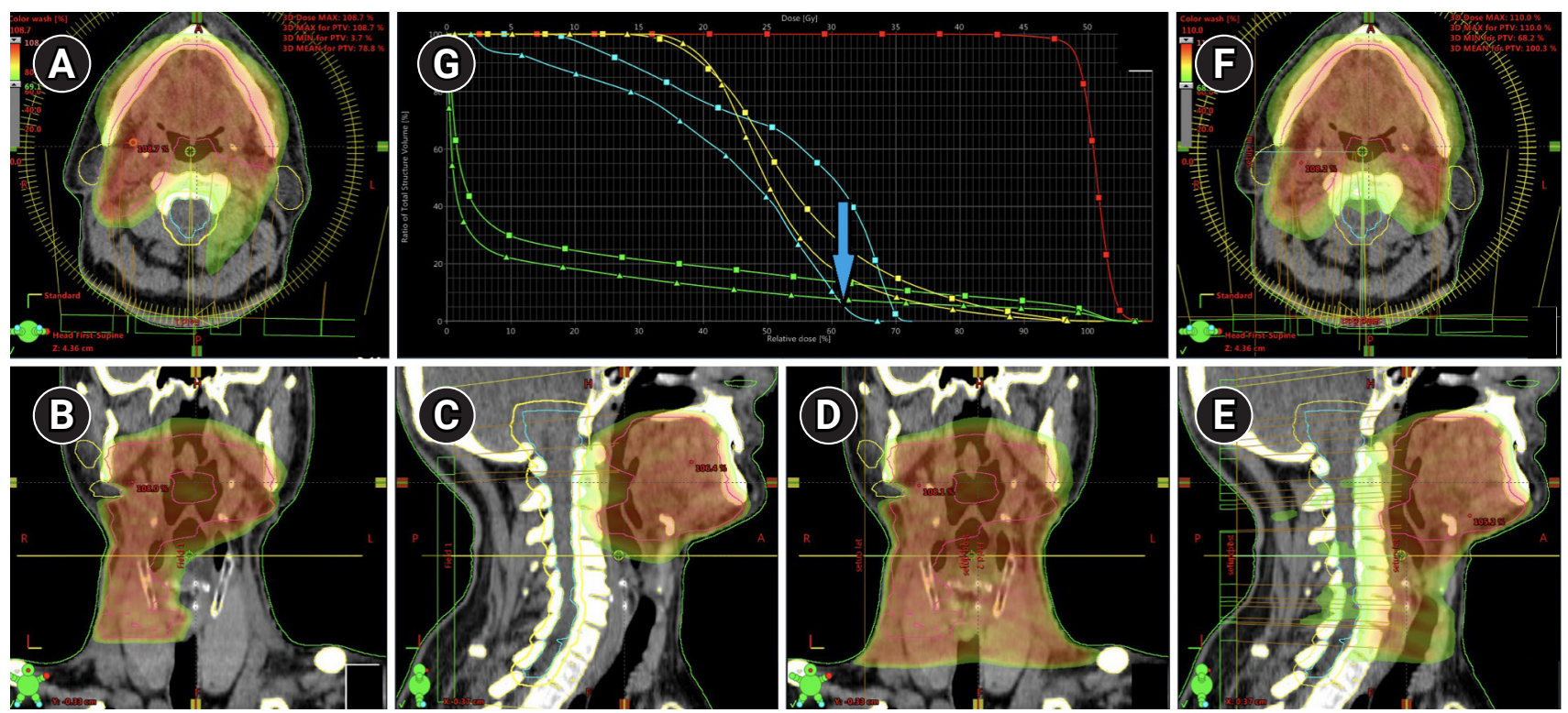

Fig. 4. Patient $C$ with a bilateral drainage from the primary lesion at SPECT-CT examination uptake of ${ }^{99 m}$ Tc-phytate was detected in lymph nodes of the I-III levels on the side of the tumor, and in lymph nodes of the II level on the contralateral side. Treatment plan for "lymph flow guided" radiotherapy of patient $\mathrm{C}$ on dose distribution in (A) axial, (B) coronal, and (C) sagittal projections. Dose distribution for a standard bilateral irradiation of neck lymph nodes presented in (D) axial, (E) coronal, and (F) sagittal projections. (G) Analysis of dose volume histograms demonstrated small reduction of the doses absorbed by the contralateral parotid gland (yellow lines), cervical spinal cord (blue arrow) with a simultaneous reduction of the total amount of irradiated tissues (green lines). SPECT-CT, single photon emission computed tomography-computed tomography.

drainage the volume of irradiated normal tissues can be reduced more than two times. Daisne et al. [12] come to the same conclusion: irradiation of LNs according the topography of SLNs can reduce the planning treatment volume by a factor of 2 . Jellema et al. [20] calculated that this dramatic reduction of the irradiated volume could decrease the frequency of dysphagia and xerostomia from $18.1 \%$ to $6.8 \%$ and from $14.5 \%$ to $1 \%$, respectively. de Veij Mestdagh et al. [21] evaluate the dosimetric benefits of SPECT-CT guided irradiation of neck LNs and the probability of normal tissue complications. The authors concluded that the unilateral irradiation of neck LNs in patients with a unilateral lymphatic drainage reduces the frequency and severity of dysphagia, hypothyroidism and laryngeal edema. In particular, they calculated that the probability of xerostomia would be reduced from $43.3 \%$ to $22 \%$, dysphagia from $18.1 \%$ to $6.8 \%$, hypothyroidism from $44.4 \%$ to $20.2 \%$, and laryngeal edema from $24 \%$ to $7 \%$.

The possibility for further reduction of the LN clinical target volume when it is limited by irradiation of LNs levels that contain radioactive ("hot") LNs looks very attractive but must be implemented in clinical practice with caution. According to the SENT multicenter prospective study that included 434 patients with cT12N0M0 squamous cell oropharyngeal cancer, $96 \%$ of the metastatic LNs were located at the same levels as the SLNs visualized with SPECT-CT, and in most cases those were the nodes localized at lev- els I-III [22]. Miura et al. [23] report that in 57 patients with cT23 NOMO squamous cell oropharyngeal cancer SLNs were detected only at levels IV-V in 6\%, and never had signs of a metastatic invasion. The SUSPECT trial [8] also reports that in patients with oral cancer SLNs in most cases are localized at levels II-III. Sagheb et al. [24] point out that in patients with cT1-2N0M0 oral tongue cancer the risk of occult LNs metastases is around 30\%. However, they did not find any LN involvement distally to the level containing SLNs. These data indicate that in 13 of 26 patients included in our study the radiotherapy volume can be limited by a unilateral irradiation of the LNs localized at levels I-II or I-III. However, one has to be aware that the implementation of this treatment strategy may well lead to the increase of regional recurrences and therefore needs to be evaluated during the ongoing and planning prospective trials.

Our study has several important limitations. The main is that radiotherapy is usually indicated in patients with metastatic LNs. In our study the analysis was performed in patients without clinical signs of $L N$ involvement. It would be important to validate the obtained data on LN positive population. Next, according to the institute treatment guidelines most of the patients underwent a unilateral LNs dissection, and the risk of contralateral LNs involvement was mainly assessed on the basis of a 2-year follow-up which can be insufficient for a confident conclusion. Nevertheless, we can make some important conclusions. (1) The unilateral lymph flow 
from the primary lesion strongly correlated with lateral localization of this lesion. (2) In patients with oral tongue cancer and unilateral SPECT-CT localization of SLNs there was no case of contralateral metastases. (3) The histologic data and results of the follow-up demonstrate a high correlation between the levels containing SLNs and the topography of metastatic LNs. These data support the assumption that the lymph flow guided strategy is a reliable means of personalizing radiotherapy volumes. (4) It was shown that the reduction of the clinical target volume to neck levels containing SLNs could significantly reduce the cumulative volume of irradiated normal tissues and the mean dose to the critical organs at risk.

\section{Conflict of Interest}

No potential conflict of interest relevant to this article was reported.

\section{References}

1. Chen MM, Harris JP, Hara W, Sirjani D, Divi V. Association of postoperative radiotherapy with survival in patients with $\mathrm{N} 1$ oral cavity and oropharyngeal squamous cell carcinoma. JAMA Otolaryngol Head Neck Surg 2016;142:1224-30.

2. Kerawala C, Roques T, Jeannon JP, Bisase B. Oral cavity and lip cancer: United Kingdom National Multidisciplinary Guidelines. J Laryngol Otol 2016;130(S2):S83-9.

3. de Bondt RB, Nelemans PJ, Hofman PA, et al. Detection of lymph node metastases in head and neck cancer: a meta-analysis comparing US, USgFNAC, CT and MR imaging. Eur J Radiol 2007;64: 266-72.

4. Genden EM, Ferlito A, Silver $\mathrm{CE}_{\text {, }}$ et al. Contemporary management of cancer of the oral cavity. Eur Arch Otorhinolaryngol 2010;267:1001-17.

5. Goel R, Moore W, Sumer B, Khan S, Sher D, Subramaniam RM. Clinical practice in PET/CT for the management of head and neck squamous cell cancer. AJR Am J Roentgenol 2017;209:289-303.

6. Zhang H, Seikaly H, Biron VL, Jeffery CC. Utility of PET-CT in detecting nodal metastasis in cNO early stage oral cavity squamous cell carcinoma. Oral Oncol 2018;80:89-92.

7. Al-Mamgani A, Verheij M, van den Brekel MW. Elective unilateral nodal irradiation in head and neck squamous cell carcinoma: a paradigm shift. Eur J Cancer 2017;82:1-5.

8. de Veij Mestdagh PD, Jonker MC, Vogel WV, et al. SPECT/ CT-guided lymph drainage mapping for the planning of unilateral elective nodal irradiation in head and neck squamous cell carcinoma. Eur Arch Otorhinolaryngol 2018;275:2135-44.

9. Neiweg OE. The sentinel lymph node concept in oncologic sur- gery. In : Mariani G, Vidal-Sicart S, Valdes Olmos RA, editors. Atlas of lymphoscintigraphy and sentinal node mapping: a pictorial case-based approach. 2nd ed. Cham, Switzerland: Springer; 2020, p. 143-9.

10. Krengli M, Ballare A, Cannillo $B$, et al. Potential advantage of studying the lymphatic drainage by sentinel node technique and SPECT-CT image fusion for pelvic irradiation of prostate cancer. Int J Radiat Oncol Biol Phys 2006;66:1100-4.

11. Novikov SN, Kanaev SV, Semiglazov VF, Jukova LA, Krzhivitckiy PI. Comparison of two treatment strategies for irradiation of regional lymph nodes in patients with breast cancer: lymph flow guided portals versus standard radiation fields. Rep Pract Oncol Radiother 2014;20:27-31.

12. Daisne JF, Installe J, Bihin B, et al. SPECT/CT Iymphoscintigraphy of sentinel node(s) for superselective prophylactic irradiation of the neck in cNO head and neck cancer patients: a prospective phase I feasibility study. Radiat Oncol 2014;9:121.

13. Novikov SN, Krzhivitskii PI, Kanaev SV, et al. Lymph flow guided irradiation of regional lymph nodes in patients with cervical cancer: preliminary analysis of scintigraphic data. Rep Pract Oncol Radiother 2018;23:503-9.

14. Garrel R, Poissonnet G, Temam S, Dolivet G, Fakhry N, de Raucourt D. Review of sentinel node procedure in cNO head and neck squamous cell carcinomas: guidelines from the French evaluation cooperative subgroup of GETTEC. Eur Ann Otorhinolaryngol Head Neck Dis 2017;134:89-93.

15. Gregoire V, Ang K, Budach W, et al. Delineation of the neck node levels for head and neck tumors: a 2013 update: DAHANCA, EORTC, HKNPCSG, NCIC CTG, NCRI, RTOG, TROG consensus guidelines. Radiother Oncol 2014;110:172-81.

16. D'Cruz AK, Vaish $R$, Kapre $N$, et al. Elective versus therapeutic neck dissection in node-negative oral cancer. N Engl J Med 2015; 373:521-9.

17. Farmer RW, McCall L, Civantos FJ, et al. Lymphatic drainage patterns in oral squamous cell carcinoma: findings of the ACOSOG Z0360 (Alliance) study. Otolaryngol Head Neck Surg 2015;152: 673-7.

18. Schilling C, Stoeckli SJ, Haerle SK, et al. Sentinel European Node Trial (SENT): 3 -year results of sentinel node biopsy in oral cancer. Eur J Cancer 2015;51:2777-84.

19. Singh B, Nair S, Nair D, Patil A, Chaturvedi P, D'Cruz AK. Ipsilateral neck nodal status as predictor of contralateral nodal metastasis in carcinoma of tongue crossing the midline. Head Neck 2013;35:649-52.

20. Jellema AP, Slotman BJ, Doornaert $P$, Leemans CR, Langendijk JA. Unilateral versus bilateral irradiation in squamous cell head and neck cancer in relation to patient-rated xerostomia and sticky 
saliva. Radiother Oncol 2007;85:83-9.

21. de Veij Mestdagh PD, Walraven I, Vogel WV, et al. SPECT/CT-guided elective nodal irradiation for head and neck cancer is oncologically safe and less toxic: a potentially practice-changing approach. Radiother Oncol 2020;147:56-63.

22. Tartaglione G, Stoeckli SJ, de Bree $R$, et al. Sentinel node in oral cancer: the nuclear medicine aspects: a survey from the Sentinel European Node Trial. Clin Nucl Med 2016;41:534-42.
23. Miura $K$, Hirakawa $H$, Uemura $H$, et al. Sentinel node biopsy for oral cancer: a prospective multicenter phase II trial. Auris Nasus Larynx 2017;44:319-26.

24. Sagheb K, Sagheb K, Rahimi-Nedjat R, Taylor K, Al-Nawas B, Walter $C$. Sentinel lymph node biopsy in T1/T2 squamous cell carcinomas of the tongue: a prospective study. Oncol Lett 2016; 11:600-4. 